\title{
Probing Single-Photon Ionization on the Attosecond Time Scale
}

\author{
K. Klünder, ${ }^{1}$ J. M. Dahlström, ${ }^{1}$ M. Gisselbrecht, ${ }^{1}$ T. Fordell, ${ }^{1}$ M. Swoboda, ${ }^{1}$ D. Guénot,,${ }^{1}$ P. Johnsson, ${ }^{1}$ J. Caillat, ${ }^{2}$ \\ J. Mauritsson, ${ }^{1}$ A. Maquet, ${ }^{2}$ R. Taïeb, ${ }^{2}$ and A. L'Huillier ${ }^{1, *}$ \\ ${ }^{1}$ Department of Physics, Lund University, P.O. Box 118, 22100 Lund, Sweden \\ ${ }^{2}$ Laboratoire de Chimie Physique-Matière et Rayonnement, Université Pierre et Marie Curie, 11, Rue Pierre et Marie Curie, \\ 75231 Paris Cedex, 05, France
}

(Received 15 December 2010; published 5 April 2011; publisher error corrected 14 April 2011)

\begin{abstract}
We study photoionization of argon atoms excited by attosecond pulses using an interferometric measurement technique. We measure the difference in time delays between electrons emitted from the $3 s^{2}$ and from the $3 p^{6}$ shell, at different excitation energies ranging from 32 to $42 \mathrm{eV}$. The determination of photoemission time delays requires taking into account the measurement process, involving the interaction with a probing infrared field. This contribution can be estimated using a universal formula and is found to account for a substantial fraction of the measured delay.
\end{abstract}

PACS numbers: $32.80 . \mathrm{Rm}, 32.80 . \mathrm{Qk}, 42.65 . \mathrm{Ky}$

The interaction of light with matter is an essential process in nature and, in particular, the photoelectric effect has been studied during decades using synchrotron radiation [1]. The development of ultrashort light pulses in the attosecond range allows scientists to tackle temporal aspects of electron transitions in atoms, molecules, and more complex systems. Cavalieri et al. [2] investigated photoemission from the valence and the conduction band in tungsten crystals using single attosecond pulses and an infrared (ir) probing field through the streaking technique [3]. Recently, Schultze et al. [4] implemented the same technique to study photoemission from the $2 s^{2}$ and $2 p^{6}$ shells in neon at a pulse energy of $100 \mathrm{eV}$. They measured a difference in photoemission time delays equal to 21 as, a value which is significantly larger than the expected theoretical value, as further discussed in a series of theoretical articles [5-8].

In this Letter, we examine photoemission of electrons from the $3 s^{2}$ and $3 p^{6}$ shells in argon. Our method uses a frequency comb of high-order harmonics with photon energies varying from 32 to $42 \mathrm{eV}$ for the photoionization and a weak ir field for probing the outgoing electrons. It is based on interferometry and presents analogies with coherent control schemes used for phase measurements close to resonant states [9-11]. Here we explore single photoionization in the threshold region for the $3 s^{2}$ shell, where one expects large variation in photoemission times. The measurement shows a delay between the ionization from the $3 s^{2}$ and $3 p^{6}$ shells which varies with photon energy. We investigate the influence of the interaction with the weak ir field, which is needed to do the interferometric measurement and get the temporal information. Probing the outgoing electron wave packet (EWP), even with a weak ir field, affects electron motion and therefore the measured delay. Fortunately, this effect can be analytically calculated and takes a universal form, that allows us to disentangle the different effects and gives us access to the single-photon ionization time, also called Wigner time $[12,13]$.
The basic principle of our experiment is shown in Fig. 1. We ionize argon using a comb of high-order harmonics. With a central frequency of the harmonic comb above the binding energy of the $3 s$ shell we simultaneously create two independent EWPs, one originating from the $3 s^{2}$ and one from the $3 p^{6}$ shell. The presence of a fraction of the fundamental laser field with frequency $\omega$ induces the formation of sideband peaks due to two-photon transitions including absorption or emission of an ir photon $[14,15]$. Two different and interfering quantum paths involving consecutive harmonics lead to the same sideband (see Fig. 1). When changing the delay $\tau$ between the harmonic comb and the laser field, the sideband signal from a given shell is modulated as [16]

$$
S(\tau)=\alpha+\beta \cos \left[2 \omega\left(\tau-\tau_{\mathrm{A}}-\tau_{\mathrm{I}}\right)\right],
$$

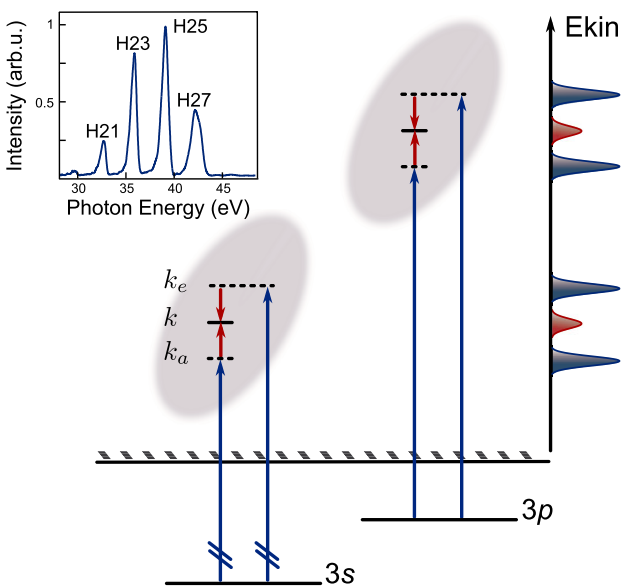

FIG. 1 (color online). Principle of the measurement. Two EWPs originating from different shells are simultaneously created using the same comb of high-order harmonics. The outgoing EWPs are further probed with a weak ir field. For simplicity only two harmonics are indicated. Also shown is the experimental harmonic spectrum used. 
where $\alpha, \beta$ are two constants independent of $\tau$. The term $\tau_{\mathrm{A}}$ is proportional to the difference in phase between consecutive harmonics and describes the group delay of the attosecond pulses, while $\tau_{\text {I }}$ represents the atomic delay due to the two-photon ionization process [17]. As we will show below $\tau_{\mathrm{I}}$ can be connected to the Wigner time delay $\tau_{\mathrm{W}}$ for the single-photon ionization. The knowledge of $\tau_{\mathrm{A}}$ as well as of the absolute value of the delay $\tau$ would enable us to determine $\tau_{\text {I }}$ directly. However, these variables are difficult to obtain separately. The simultaneous measurement of the two EWPs allows us to cancel the influence of the attosecond group delay $\tau_{\mathrm{A}}$ and to determine $\tau_{\mathrm{I}}(3 s)-\tau_{\mathrm{I}}(3 p)$ at the same photon energy, i.e., at kinetic energies separated by the difference in binding energy between the two shells $(13.5 \mathrm{eV})$.

Our experiments were performed with a $800 \mathrm{~nm}, 30 \mathrm{fs}$ titanium-sapphire laser system [18]. High-order harmonics were generated in a pulsed Ar gas cell and spatially filtered using a small aperture [19]. We used a $0.2 \mu \mathrm{m}$ thick chromium thin film to select a $10 \mathrm{eV}$-broad spectral window corresponding to harmonic 21 to 27 at $38 \mathrm{eV}$ central energy (see Fig. 1). This filter was chosen to separate the wave packets emitted from the $3 s$ and $3 p$ shells in energy. The comb of four phase-locked harmonics, corresponding to a train of attosecond pulses with a 450 as duration, was focused by a toroidal mirror into the sensitive region of a magnetic bottle electron spectrometer containing a diffusive Ar gas jet. Part of the laser field was extracted prior to the high-order harmonic generation and recombined collinearly with the harmonics with a variable time delay $\tau$. The precision of our measurement does not depend on the duration of the attosecond extreme-ultraviolet (xuv) pulses but on the interferometric stability of our experiment, estimated to be 50 as.

Figures 2(a) and 2(b) present electron spectra as a function of the delay $\tau$ between the xuv and the ir pulses. The low-energy spectrum in Fig. 2(a) shows electron peaks at energies corresponding to single-photon ionization from the $3 s$ shell by the harmonics and additional sideband peaks due to two-photon transitions. The high-energy part of the spectrum shown in Fig. 2(b) presents the corresponding photoelectron spectra for $3 p$ ionization. Although simultaneously recorded the results are presented separately due to the unequal signal strength caused by the difference in cross section and detector sensitivity (note the different color scales). For both channels the sideband signal oscillates, allowing us to extract the delay by Fourier transform along the time axis for a weak ir field [20]. The ir intensity was estimated to be well below $10^{12} \mathrm{~W} \mathrm{~cm}^{-2}$. Figure 2(c) presents the delays obtained for the scan shown in (a) and (b), corrected for the influence of the Cr filter, which is positively dispersive in this region [21]. The variation in delay reflects mainly the positive chirp of the attosecond pulses. The main experimental result of the present work is the significant offset
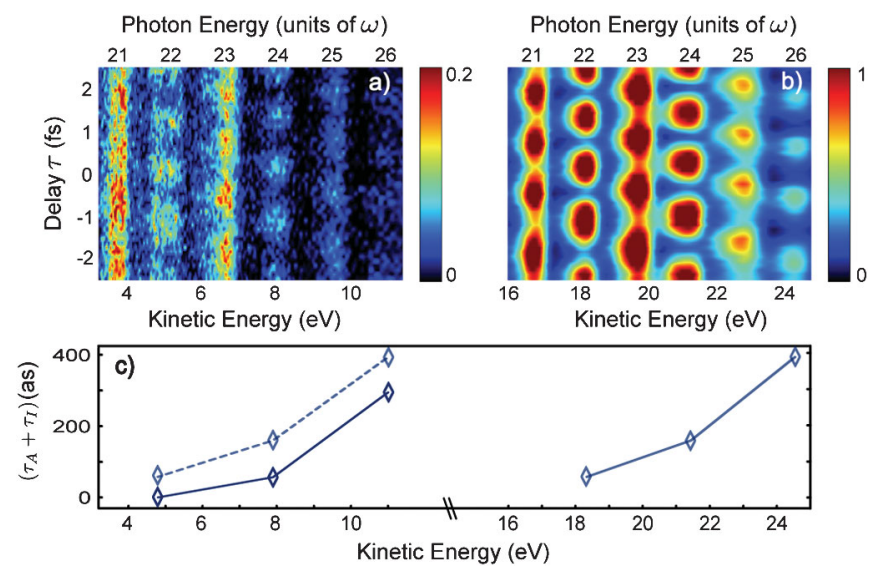

FIG. 2 (color). Energy spectra as a function of delay from electrons liberated from the $3 s$ shell (a) and the $3 p$ shell (b), respectively. (c) Retrieved delays corrected for the $\mathrm{Cr}$ group delay. Also shown are the $3 p$ delays shifted down in energy for comparison with the $3 s$ delays (dashed line).

between the delays measured for the two wave packets. To emphasize this result, we show as a dashed line the $3 p$ delays shifted down in energy by $13.5 \mathrm{eV}$. Taking the difference between the measured delays at the same excitation energy allows us to eliminate the attosecond chirp and to reduce the effect of temporal drifts in the interferometer. Averaging over five independent measurements, we determine a difference in delays $\tau_{\mathrm{I}}(3 s)-\tau_{\mathrm{I}}(3 p)$ equal to $-40 \pm 10$ as for sideband $22,-110 \pm 10$ as for sideband 24 , and $-80 \pm 30$ as for sideband 26 .

To understand the meaning of these time delays, we need to establish the connection between single-photon ionization and the two-photon ionization process used in the measurement. The phase of the transition matrix element describing a single ionization process towards a final state with angular momentum $\ell$ is the scattering phase $\eta_{\ell}$, i.e., the phase accumulated by the photoelectron when escaping from the atom. Its energy derivative $\tau_{\mathrm{W}}=\hbar \partial \eta_{\ell}(\epsilon) / \partial \epsilon$ represents the "photoionization time delay" also called Wigner time delay $[12,13]$. Clearly, both $\eta_{\ell}$ and $\tau_{\mathrm{W}}$ depend on the details of the atomic potential and their computation remains a challenge for theory. Using second-order perturbation theory, the transition matrix element for two-photon ionization involving absorption of a harmonic photon $\omega_{\mathrm{H}}$ and an ir photon $\omega$ from an initial state $\varphi_{i}$ to a continuum state $\varphi_{\vec{k}}$ with asymptotic momentum $\vec{k}$ can, using atomic units, be written as

$M_{a}^{(2)}(\vec{k})=-i E_{\mathrm{L}} E_{\mathrm{H}} \lim _{\varepsilon \rightarrow 0^{+}} \oiint_{n} \frac{\left\langle\varphi_{\vec{k}}|\vec{\epsilon} \cdot \vec{r}| \varphi_{n}\right\rangle\left\langle\varphi_{n}|\vec{\epsilon} \cdot \vec{r}| \varphi_{i}\right\rangle}{\epsilon_{i}+\omega_{\mathrm{H}}-\epsilon_{n}+i \varepsilon}$.

The complex amplitudes of the laser and harmonic fields are denoted $E_{\mathrm{L}}$ and $E_{\mathrm{H}}$ and $\vec{\epsilon}$ is their common polarization vector. The energies of the initial and intermediate states are denoted $\epsilon_{i}$ and $\epsilon_{n}$, respectively. The integral sum is performed over all possible intermediate states $\varphi_{n}$. 
The index $a$ indicates that we first discuss a two-photon process with absorption of the ir photon.

We consider the channels $s \rightarrow p \rightarrow \ell$ with $\ell=s, d$. Using spherical coordinates, separating radial and angular parts, and expanding the final wave function into partial waves, the transition matrix element becomes

$$
M_{a}^{(2)}(\vec{k})=-i E_{\mathrm{L}} E_{\mathrm{H}} \sum_{\ell=0,2} C_{\ell 0} Y_{\ell 0}(\hat{k}) e^{i \eta_{\ell}(k)} T_{a}^{(2)}(k),
$$

where $Y_{\ell 0}$ is a spherical harmonic, $C_{\ell 0}$ is the corresponding angular coefficient, and $\eta_{\ell}$ is the scattering phase of the final state. The radial two-photon transition matrix element $T_{a}^{(2)}(k)$ can be expressed as $[15,17]$

$$
T_{a}^{(2)}(k)=\oint_{n} \frac{\left\langle R_{k \ell}|r| R_{n 1}\right\rangle\left\langle R_{n 1}|r| R_{i 0}\right\rangle}{\epsilon_{i}+\omega_{\mathrm{H}}-\epsilon_{n}+i \varepsilon}=\left\langle R_{k \ell}|r| \rho_{k_{a} 1}\right\rangle .
$$

In the right part of Eq. (4) we introduce the perturbed wave function $\rho_{k_{a}}$ with the wave number $k_{a}$ such that $k_{a}^{2} / 2=$ $\epsilon_{i}+\omega_{\mathrm{H}}=k^{2} / 2-\omega$ (see Fig. 1) [22]. To get an estimate of the phase of $T_{a}^{(2)}$, we consider the asymptotic behavior of the wave functions involved in Eq. (4). The perturbed wave function $\rho_{k_{a} 1}$ is an outgoing wave [23,24]

$$
\lim _{r \rightarrow \infty} \rho_{k_{a} 1}(r) \propto e^{i\left[k_{a} r-1 / 2 \pi+1 /\left(k_{a}\right) \ln \left(2 k_{a} r\right)+\eta_{1}\left(k_{a}\right)\right]},
$$

while $R_{k \ell}$ is real with an asymptotic behavior:

$$
\lim _{r \rightarrow \infty} R_{k \ell}(r) \propto \sin \left[k r-\frac{\ell}{2} \pi+\frac{1}{k} \ln (2 k r)+\eta_{\ell}(k)\right] .
$$

The factor $\ell \pi / 2$ arises from the centrifugal potential, while $\ln (2 k r) / k$ is a correction due to the long-range Coulomb potential. Using Eqs. (3)-(6) we find an approximate expression for $M_{a}^{(2)}(k)$

$$
M_{a}^{(2)}(k) \propto \underbrace{e^{i \eta_{1}\left(k_{a}\right)}}_{(\mathrm{I})} \times \underbrace{\left(\frac{i}{k_{a}-k}\right)^{i z} \frac{(2 k)^{i / k}}{\left(2 k_{a}\right)^{i /\left(k_{a}\right)}} \Gamma(2+i z)}_{(\mathrm{II})},
$$

where $z=1 / k_{a}-1 / k$ and $\Gamma(z)$ is the complex gamma function. The first phase term (I) is the scattering phase of the intermediate state and identical to the phase of the corresponding one-photon ionization. The phase of term (II) can be assigned to the laser-driven transition connecting the two continuum states in the presence of the longrange Coulomb potential, $\varphi_{a}^{c c}$. It is independent of the short-range behavior of the atomic potential and therefore universal. Corrections to this approximation due to the core are expected to become important only at energies close to threshold.

The phase of the two-photon matrix element $M_{e}^{(2)}$ for the second pathway, i.e., absorption of an harmonic photon $\omega_{\mathrm{H}}$ followed by emission of an ir photon $\omega$ via an intermediate state with wave number $k_{e}^{2} / 2=k^{2} / 2+\omega$ (see Fig. 1), can be derived in a similar manner. The total interference signal is obtained by angular integration of $\left|M_{a}^{(2)}+M_{e}^{(2)}\right|^{2}$. It can be written as Eq. (1), with

$$
\tau_{\mathrm{I}}=\underbrace{\frac{\eta_{1}\left(k_{e}\right)-\eta_{1}\left(k_{a}\right)}{2 \omega}}_{\tau_{\mathrm{W}}}+\underbrace{\frac{\varphi_{e}^{c c}(k)-\varphi_{a}^{c c}(k)}{2 \omega}}_{\tau_{c c}} .
$$

This result gives an intuitive understanding of the ionization time $\tau_{\mathrm{I}}(3 s)$. It can be expressed as the sum of the Wigner time delay $\tau_{\mathrm{W}}$ for one-photon ionization $3 s \rightarrow \epsilon p$ and an additional continuum-continuum delay $\tau_{c c}$ inherent to the measuring process. This analytical derivation can be easily generalized to other ionization channels.

Figure 3 shows the delays involved in the three ionization channels $3 p \rightarrow \epsilon s$ (a), $3 p \rightarrow \epsilon d$ (b), and $3 s \rightarrow \epsilon p$ (c) in $\mathrm{Ar}$ as a function of kinetic energy. The Wigner time delay $\tau_{\mathrm{W}}$ (dashed) is obtained by taking the derivative of the scattering phase (Coulomb phase plus phase shift taken from [25]). For comparison, we also show in (d) the delays for the pathway $1 s \rightarrow \epsilon p$ in hydrogen in the same energy region, using the Coulomb phase. The continuumcontinuum delay $\tau_{c c}$ (dash-dotted) is calculated for a $800 \mathrm{~nm}$ laser wavelength and identical for all the channels and atoms. The solid line indicates $\tau_{\mathrm{I}}$ as the sum of the two contributions. The Wigner time delay variation can be nicely and intuitively interpreted. Low-energy electrons take a longer time to escape from a given shell than high-energy electrons. Furthermore, electrons escaping to a channel with higher angular momentum take a longer time than those escaping to a channel with low angular momentum because of the centrifugal barrier. The continuum-continuum delay has the opposite behavior
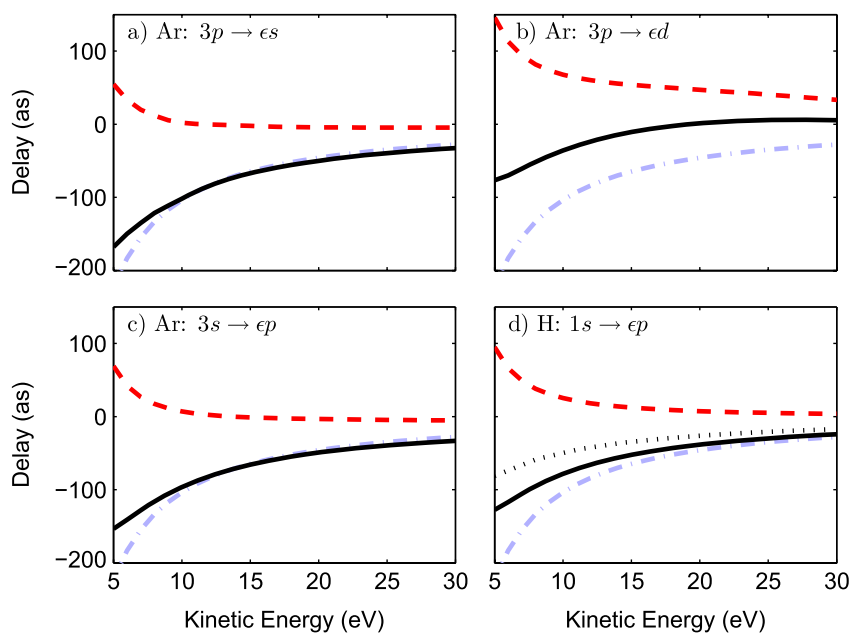

FIG. 3 (color online). Computed delays associated with the following ionization channels: (a) $3 p \rightarrow \epsilon s$, (b) $3 p \rightarrow \epsilon d$, (c) $3 s \rightarrow \epsilon p$ in $\mathrm{Ar}$, and (d) $1 s \rightarrow \epsilon p$ in $\mathrm{H}$. The dashed lines (red) are the one-photon Wigner time delays. The dash-dotted lines (blue) represent the estimated delays induced by the measurement $\tau_{c c}$. The sum of the two delays is shown as a solid line (black). The dotted line (black) in (d) is the result of an exact calculation in $\mathrm{H}$. 


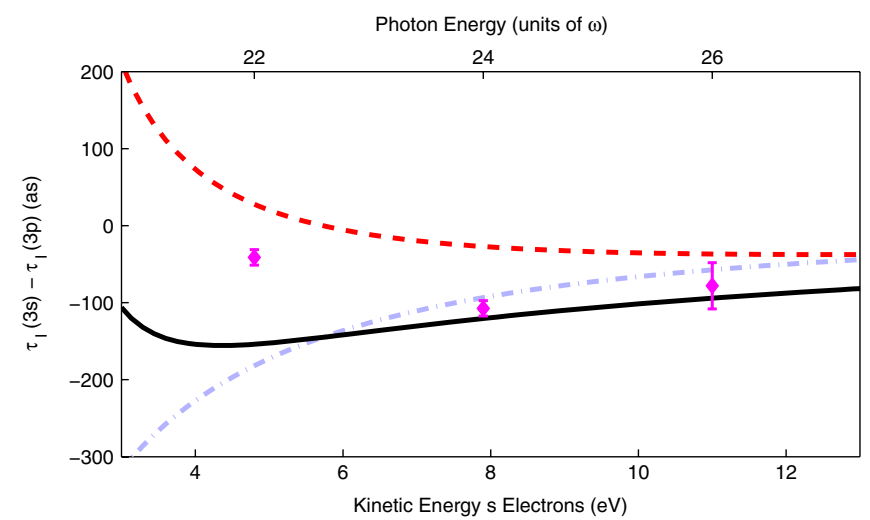

FIG. 4 (color online). Comparison between the measured delay differences for ionization of Ar from the $3 s$ and $3 p$ shells (diamonds) with calculations performed according to the approximate theory developed in this work (solid black line). Also shown is the delay expected for one-photon ionization (dashed red line) and the laser-driven continuum-continuum transition (dash-dotted blue line).

and leads to an apparent quicker escape for the low-energy electrons. Finally, we also indicate in Fig. 3(d) results from exact calculations in $\mathrm{H}$ (dotted line). The comparison between the solid and dotted lines gives an estimation of the error made in considering only the asymptotic behaviors of the perturbed and final wave functions. For the energy range considered in the present work the asymmetry parameter remains close to 2 [26], which indicates that the ionization channel $3 p \rightarrow \epsilon d$ dominates over $3 p \rightarrow \epsilon s$. Neglecting the $3 p \rightarrow \epsilon s$ channel, we calculate $\tau_{\mathrm{I}}(3 s)-\tau_{\mathrm{I}}(3 p)$ at the same excitation energy [Eq. (8)]. Figure 4 presents the approximated delays (solid line), together with the experimental results $(\diamond)$. The experimental results at the two highest energies agree well with the results of our calculation, indicating that the scattering phases [25] and our approximated continuum-continuum transition are reliable in this region. The lowest energy point, however, lies several standard deviations away from the calculated value. In this region the core may play a more important role for the continuum-continuum transition, and the Wigner time delays may differ from those calculated in [25]. In addition, the finite difference approximation to the Wigner time delay in Eq. (8) might break down in the presence of sharp resonances [27]. Using our experimental measurements combined with our estimated continuum-continuum delays, we can tentatively deduce the difference in single photoemission delays to be equal to 140 as at $34 \mathrm{eV}$ and -20 as at 37 and $40 \mathrm{eV}$.

In conclusion, we have performed experimental measurements of photoemission from the $3 s^{2}$ and $3 p^{6}$ shells in Ar, using interferometry with a weak ir field to probe the created EWPs. We identify two contributions to the measured delays: the Wigner time delay and a delay inherent to the measurement process. Both contributions are most important near threshold and vanish as the energy increases. We believe that the work presented here will stimulate further experiments, aiming at measuring photoemission delays in a variety of systems, and providing data that could be compared to advanced theoretical calculations.

We thank Stefan Haessler, Franck Lépine, and Kenneth J. Schafer for stimulating discussions. This research was supported by the Marie Curie programs ATTOCO (IEF) and ATTOFEL (ITN), the European Research Council (ALMA), the Joint Research Programme ALADIN of Laserlab-Europe II, the Swedish Foundation for Strategic Research, the Swedish Research Council, the Knut and Alice Wallenberg Foundation, and the French ANR ATTO-WAVE.

*anne.lhuillier@fysik.lth.se http://www.atto.fysik.lth.se

[1] V. Schmidt, Rep. Prog. Phys. 55, 1483 (1992).

[2] A. L. Cavalieri et al., Nature (London) 449, 1029 (2007).

[3] J. Itatani et al., Phys. Rev. Lett. 88, 173903 (2002).

[4] M. Schultze et al., Science 328, 1658 (2010).

[5] V.S. Yakovlev, J. Gagnon, N. Karpowicz, and F. Krausz, Phys. Rev. Lett. 105, 073001 (2010).

[6] J. C. Baggesen and L. B. Madsen, Phys. Rev. Lett. 104, 043602 (2010).

[7] C.-H. Zhang and U. Thumm, Phys. Rev. A 82, 043405 (2010).

[8] A.S. Kheifets and I. A. Ivanov, Phys. Rev. Lett. 105, 233002 (2010).

[9] R. Yamazaki and D. S. Elliott, Phys. Rev. Lett. 98, 053001 (2007).

[10] S. Haessler et al., Phys. Rev. A 80, 011404 (2009).

[11] M. Swoboda et al., Phys. Rev. Lett. 104, 103003 (2010).

[12] E. P. Wigner, Phys. Rev. 98, 145 (1955).

[13] F. T. Smith, Phys. Rev. 118, 349 (1960).

[14] J. M. Schins et al., J. Opt. Soc. Am. B 13, 197 (1996).

[15] V. Véniard, R. Taïeb, and A. Maquet, Phys. Rev. A 54, 721 (1996).

[16] P. M. Paul et al., Science 292, 1689 (2001).

[17] E. S. Toma and H. G. Muller, J. Phys. B 35, 3435 (2002).

[18] T. Fordell, M. Miranda, A. Persson, and A. L'Huillier, Opt. Express 17, 21091 (2009).

[19] R. López-Martens et al., Phys. Rev. Lett. 94, 033001 (2005).

[20] M. Swoboda et al., Laser Phys. 19, 1591 (2009).

[21] http://www.cxro.lbl.gov.

[22] A. Dalgarno and J.T. Lewis, Proc. R. Soc. A 233, 70 (1955).

[23] M. Aymar and M. Crance, J. Phys. B 13, L287 (1980).

[24] M. Edwards, X. Tang, and R. Shakeshaft, Phys. Rev. A 35, 3758 (1987).

[25] D. J. Kennedy and S. T. Manson, Phys. Rev. A 5, 227 (1972).

[26] R. G. Houlgate, J. B. West, K. Codling, and G. V. Marr, J. Electron Spectrosc. Relat. Phenom. 9, 205 (1976).

[27] R. P. Madden, D. L. Ederer, and K. Codling, Phys. Rev. 177, 136 (1969). 